Original Article

\title{
Immediate effects of lumbar spine patterns after neuromuscular joint facilitation on balance in stroke patients
}

\author{
Hualong Xie, PT, MS ${ }^{1)^{*}}$, Ming Huo, PT, PhD ${ }^{2}$, Qiuchen Huang, PT, $\mathrm{PhD}^{3}$, 4), \\ Danyang Zhou, PT $^{5)}$, Shan Liu, PT, MS ${ }^{1)}$, Suli Yu, PT ${ }^{5}$, Lei Chen, PT $^{5}$, \\ Ko Onoda, PT, $\mathrm{PhD}^{1)}$, Hitoshi Maruyama, PT, $\mathrm{PhD}^{1)}$ \\ 1) Department of Physical Therapy, International University of Health and Welfare: \\ 2600-1 Kitakanemaru Otawara city, Tochigi 324-8501, Japan \\ 2) Department of Physical Therapy, Faculty of Medical Health, Himeji Dokkyo University, Japan \\ 3) School of Rehabilitation Medicine, Capital Medical University, China \\ 4) China Rehabilitation Research Center, China \\ 5) Jilin Province Power Hospital, China
}

\begin{abstract}
Purpose] The present study investigated changes in the balance function of stroke patients after neuromuscular joint facilitation treatment. [Participants and Methods] Fourteen stroke patients were randomly subjected to neuromuscular joint facilitation intervention (neuromuscular joint facilitation intervention group) and no intervention (control group), with a 1-day interval between treatments. The interventions were performed consecutively. The order of interventions was completely randomized. Before and after one neuromuscular joint facilitation and control intervention, the functional reach test, and body sway were measured. [Results] Functional reach test values were significantly increased and peripheral area was significantly reduced in the neuromuscular joint facilitation intervention group than in the control group. [Conclusion] These results suggest that neuromuscular joint facilitation of the trunk has an immediate effect on balance and function in stroke patients.

Key words: Neuromuscular joint facilitation, Stroke patients, Balance function
\end{abstract}

(This article was submitted Jun. 29, 2019, and was accepted Sep. 19, 2019)

\section{INTRODUCTION}

Symptoms of partial paralysis caused by the onset of stroke, somatosensory dysfunction, and muscle weakness are manifested as degraded balance function, deteriorated walking ability, and restricted movement in the daily life of affected individuals $^{1,2}$. Evaluation and treatment of the trunk function in early stage after the onset of stroke can help comprehensively assess the activities of daily life (ADL) of stroke patients so that they can be discharged early from the hospital. Therefore, improving trunk function of the stroke patients is crucial to improve their balancing ability, walking ability, and ADL. Hence, it is necessary to establish effective rehabilitation of trunk function in stroke rehabilitation ${ }^{3)}$.

Proprioceptive neuromuscular facilitation (PNF) can improve lower-limb muscle strength and gait function, while neuromuscular joint facilitation (NJF) is a novel kinesiology-based therapeutic exercise that can increase strength, flexibility, and range of motion. By integrating the facilitation element of PNF and the joint composition movement, NJF can improve the joint movements through passive, active, and resistance exercises ${ }^{4}$. Previous studies suggest that performing NJF in the knee joint can strengthen the muscle and shorten the integrated electromyography amplitude reaction time in healthy adults ${ }^{5,6}$. In addition, NJF in the hip joints can improve the standing balance function in healthy young people ${ }^{7)}$.

*Corresponding author. Hualong Xie (E-mail: longyuzhektt@126.com)

(C2019 The Society of Physical Therapy Science. Published by IPEC Inc.

(c) (i) $(-)$ This is an open-access article distributed under the terms of the Creative Commons Attribution Non-Commercial No Deriva-

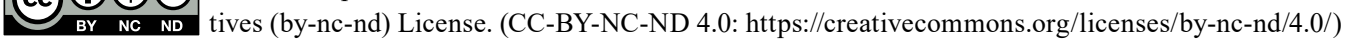


The present study investigated the change in the balance function of stroke patients after NJF treatment.

\section{PARTICIPANTS AND METHODS}

The participants were 14 stroke patients (10 males and 4 females; hemiplegia (left): 8 and hemiplegia (right): 6; Brunnstrom stage: III in 4 participants, IV in 5 participants, and V in 5 participants; mean age, $52.3 \pm 10.5$ years; mean height, $167.0 \pm$ $8.3 \mathrm{~cm}$; mean weight, $70.1 \pm 10.4 \mathrm{~kg}$ ). An NJF intervention (NJF group) and no intervention (control group) were performed with a 1-day interval among all participants. The interventions were performed one after the other. The order of interventions was completely randomized. All participants were screened before beginning the study by asking them to fill out a medical history questionnaire. Patients with first-episode stroke who could walk alone without crutches were eligible for inclusion in the study. The exclusion criteria were instability of the general condition and presence of neurological symptoms, osteoarthritis, or cognitive and psychiatric disorders. Patients with brainstem or bilateral lesions or exercise-restricted respiratory and circulatory diseases were also excluded. All participants provided informed consent for participation in the study. All experimental procedures in this study were reviewed and approved by the Ethical Review Committee of the International University of Health and Welfare.

Before and after one intervention of NJF and control, the functional reach test (FRT) was performed and body sway (BS) was measured. The FRT is an index of the dynamic standing position balance. The FRT was performed using the "yardstick" method reported by Duncan et $\mathrm{al}^{8}$. . The participants removed their shoes and socks and stood in an upright position with their feet separated at shoulder-width distance. They lifted their non-hemiplegic side arm horizontally at approximately $90^{\circ}$ with extended elbow and grip hand at the starting position in the FRT. Subsequently, they were asked to reach out as far as they could without losing their balance or taking a step. The FRT was performed twice, and the mean value of the two measurements was used in the analysis.

To measure the BS, the Zebris FDM-T System (Zebris Medical Gmb, Germany) was used. The measurement was recorded with the participants not wearing their shoes and standing in a double-legged position on the barycenter with their eyes open; the measurement was conducted for 30 seconds. The total lengths and peripheral area, an index of the static standing position balance, were measured.

Four lumbar spine patterns of the NJF were used, including the pelvic anterior elevation-proximal fixation pattern (Fig. 1), pelvic posterior depression-proximal fixation pattern (Fig. 2), pelvic anterior depression-proximal fixation pattern (Fig. 3), and pelvic posterior elevation-proximal fixation pattern (Fig. 4). Each pattern was randomly performed five times as passive and resistance exercises for the right and left sides of the participants. In the NJF intervention group, both the proximal and distal resistance exercises were performed. The control group rested for 10 minutes. The participants were instructed by the same physical therapist in both groups to avoid individual variations in treatment.
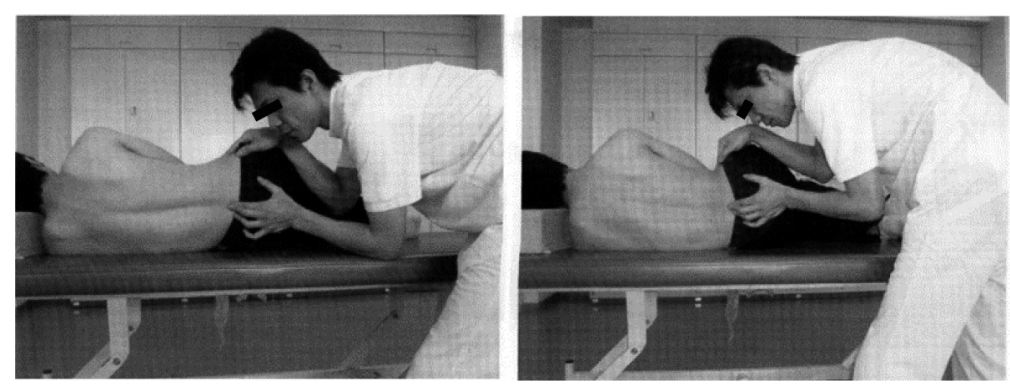

Fig. 1. Pelvic anterior elevation-proximal fixation pattern.
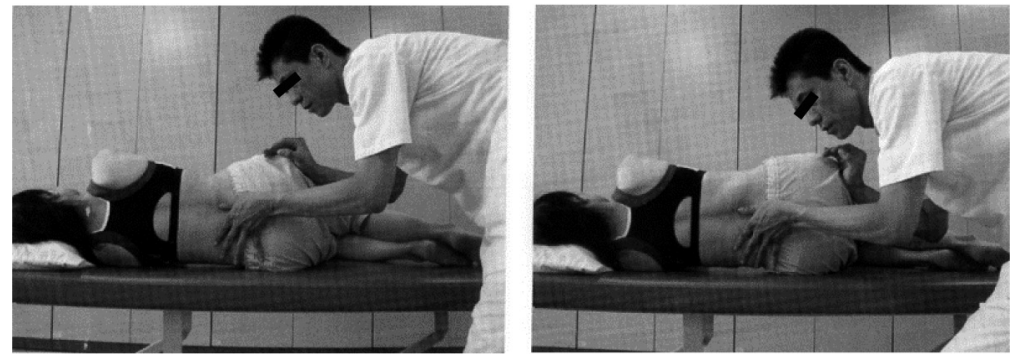

Fig. 2. Pelvic posterior depression-proximal fixation pattern. 
Two-way repeated-measures analysis of variance (ANOVA) was used to test for statistically significant differences between the intervention and control groups. If any significant interaction was found, the paired t-test was performed to compare the outcome indicators before and after the intervention. The data were analyzed using SPSS Ver. 23.0 for Windows. The level of statistical significance was set at 0.05 .

\section{RESULTS}

There were no significant differences in the participants' characteristics between the NJF and control groups or in any of the measurements before the intervention (Table 1).

Two-way ANOVA revealed significant changes in the FRT value and peripheral area between the groups, indicating that the change was different between the groups. The FRT values significantly increased and peripheral area was reduced after the NJF intervention.

\section{DISCUSSION}

The study investigated the effects of an NJF treatment on balance in stroke patients. Compared with those in the control group, the FRT values were significantly increased and the peripheral area was significantly reduced in the NJF group.

Proximal fixation of the lumbar vertebrae was performed in the NJF group. Mobilization of the lumbar facet occurred as a result of proximal fixation. The function of the lumbar facet and trunk function was improved by the NJF intervention ${ }^{9}$. NJF integrates the facilitation element of PNF and the joint composition movement. During NJF resistance exercise, the muscle
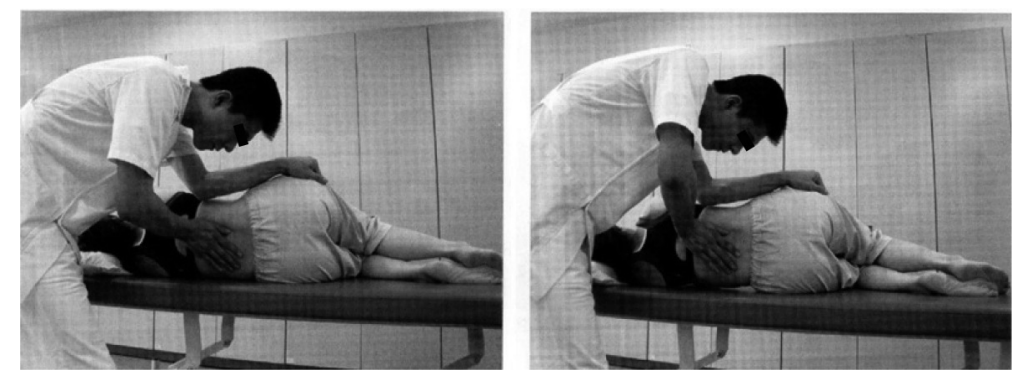

Fig. 3. Pelvic anterior depression-proximal fixation pattern.
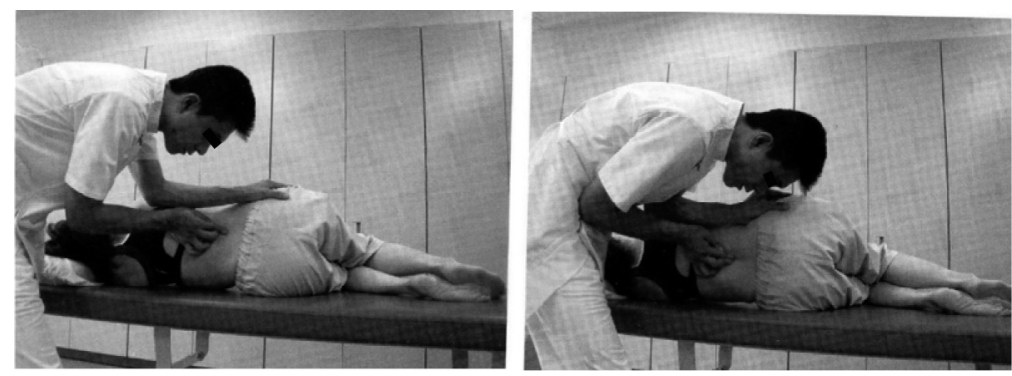

Fig. 4. Pelvic posterior elevation-proximal fixation pattern.

Table 1. Comparison of the before and after intervention values

\begin{tabular}{lcccc}
\hline & \multicolumn{2}{c}{ NJF } & \multicolumn{2}{c}{ Control } \\
\cline { 2 - 5 } & Before intervention & After intervention & Before intervention & After intervention \\
\hline FRT $(\mathrm{cm})$ & $21.6 \pm 6.2$ & $23.7 \pm 7.7^{*}$ & $21.4 \pm 5.6$ & $22.2 \pm 6.7$ \\
Total length from BS $(\mathrm{mm})$ & $849.0 \pm 194.5$ & $838.7 \pm 180.7$ & $847.9 \pm 186.4$ & $833.6 \pm 168.4$ \\
Peripheral area from BS $\left(\mathrm{mm}^{2}\right)$ & $152.2 \pm 151.6$ & $103.3 \pm 110.2^{*}$ & $118.0 \pm 100.9$ & $108.5 \pm 92.0$ \\
\hline
\end{tabular}

Data are presented as mean \pm standard deviation.

NJF: neuromuscular joint facilitation intervention; Control: no intervention; FRT: functional reach test; BS: body sway.

$* \mathrm{p}<0.05$ indicates significant difference between the groups 
strength of the trunk increased. A previous study analyzed trunk muscle strength in relation to balance and functional disability in unihemispheric stroke patients and reported trunk flexion and extension muscle weakness in these patients, which could interfere with balance, stability, and functional disability ${ }^{10)}$.

Our results suggest that an NJF intervention with proximal resistance training can be used as a novel form of exercise to improve balance function in stroke patients.

\section{Conflict of interest}

The authors declare that there are no conflicts of interest related to this work.

\section{REFERENCES}

1) Bonan IV, Colle FM, Guichard JP, et al.: Reliance on visual information after stroke. Part I: Balance on dynamic posturography. Arch Phys Med Rehabil, 2004, 85: 268-273. [Medline] [CrossRef]

2) Patterson KK, Parafianowicz I, Danells CJ, et al.: Gait asymmetry in community-ambulating stroke survivors. Arch Phys Med Rehabil, 2008, 89: 304-310. [Medline] [CrossRef]

3) Hsieh CL, Sheu CF, Hsueh IP, et al.: Trunk control as an early predictor of comprehensive activities of daily living function in stroke patients. Stroke, 2002, 33: 2626-2630. [Medline] [CrossRef]

4) Huo M: Neuromuscular joint facilitation. Tokyo: Ipec Press, 2010, p 3 (in Japanese).

5) Ge M, Huo M, Maruyama H: The immediate effect of neuromuscular joint facilitation (NJF) treatment on knee muscle force. J Phys Ther Sci, 2012, 24: 157-159. [CrossRef]

6) Huo M, Ge M, Li D, et al.: Effects of neuromuscular joint facilitation on electromechanical reaction time of rectus femoris. J Phys Ther Sci, 2012, 24: 55-57. [CrossRef]

7) Onoda K, Huo M, Maruyama H: The immediate effect of neuromuscular joint facilitation (NJF) treatment on the standing balance in younger persons. J Phys Ther Sci, 2015, 27: 1481-1483. [Medline] [CrossRef]

8) Duncan PW, Weiner DK, Chandler J, et al.: Functional reach: a new clinical measure of balance. J Gerontol, 1990, 45: M192-M197. [Medline] [CrossRef]

9) Huo M, Maruyama H, Kaneko T, et al.: The immediate effect of lumbar spine patterns of neuromuscular joint facilitation in young amateur baseball players. J Phys Ther Sci, 2013, 25: 1523-1524. [Medline] [CrossRef]

10) Karatas M, Cetin N, Bayramoglu M, et al.: Trunk muscle strength in relation to balance and functional disability in unihemispheric stroke patients. Am J Phys Med Rehabil, 2004, 83: 81-87. [Medline] [CrossRef] 